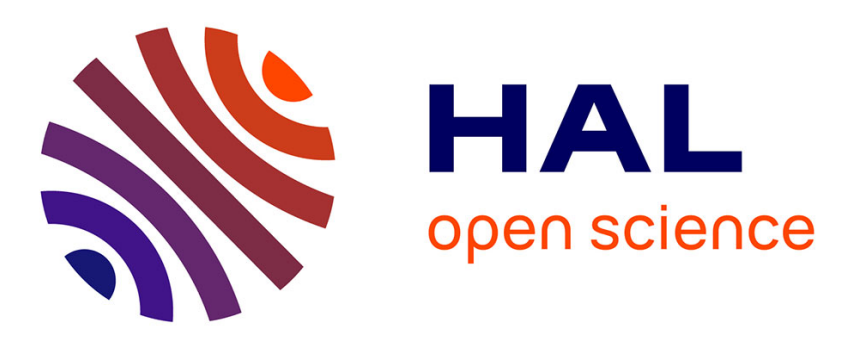

\title{
Porous Bioceramics Produced by Impregnation of 3D-Printed Wax Mold: Ceramic Architectural Control and Process Limitations
}

Baptiste Charbonnier, Coralie Laurent, Gilles Blanc, Olivier Valfort, David Marchat

\section{- To cite this version:}

Baptiste Charbonnier, Coralie Laurent, Gilles Blanc, Olivier Valfort, David Marchat. Porous Bioceramics Produced by Impregnation of 3D-Printed Wax Mold: Ceramic Architectural Control and Process Limitations. Advanced Engineering Materials, 2016, 18 (10), pp.1728-1736. 10.1002/adem.201600308 . hal-01347084

\section{HAL Id: hal-01347084 \\ https://hal.science/hal-01347084}

Submitted on 15 Nov 2016

HAL is a multi-disciplinary open access archive for the deposit and dissemination of scientific research documents, whether they are published or not. The documents may come from teaching and research institutions in France or abroad, or from public or private research centers.
L'archive ouverte pluridisciplinaire HAL, est destinée au dépôt et à la diffusion de documents scientifiques de niveau recherche, publiés ou non, émanant des établissements d'enseignement et de recherche français ou étrangers, des laboratoires publics ou privés. 
Porous Bioceramics Produced by Impregnation of 3D-Printed Wax Mold: Ceramic Architectural Control and Process Limitations

Baptiste Charbonnier; Coralie Laurent; Gilles Blanc; Olivier Valfort; David Marchat *

\author{
* Ph.D. D. Marchat \\ Ecole Nationale Supérieure des Mines de Saint-Etienne \\ CIS-EMSE, INSERM U1059, SAINBIOSE, IFRESIS \\ SPIN-EMSE, LGF UMR 5307, ProPICE \\ F-42023 Saint-Etienne, France \\ Email: marchat@emse.fr
}

\begin{abstract}
Current architectural and production limitations prevent the manufacturing standardization, design, and reproducibility of bioceramics. Such limitations have hindered advances in bone tissue engineering and regeneration. In this context, we propose a bioceramic manufacturing process based on the impregnation of wax molds produced by additive manufacturing. Hydroxyapatite (HA) bioceramics with custom architectures were fabricated to evaluate the reliability, robustness and limitations of this process. Results indicate that this process preserves phase biocompatibility, permits homogeneous shrinkage of the biomaterial during heat treatment, and allows reproducible and precise manufacturing of custom architectures (e.g., $5 \mu \mathrm{m}$ widths and high cusps for channels of $200 \mu \mathrm{m}$ with a $6 \mu \mathrm{m}$ printer resolution). Therefore, the architectural opportunities offered by this process widely exceed its architectural limitations, indicating its potential for both therapeutic applications and fundamental research.
\end{abstract}

Keywords: Bioceramic; Calcium phosphates; Additive manufacturing; Architecture 


\section{Introduction}

Calcium phosphate (CaP) bioceramics have been used for years for therapeutic purposes ${ }^{[1]}$ and for fundamental research. ${ }^{[2]}$ The first type of applications is the development of bone substitutes for regenerative medicine (e.g., repair of small bone defects). The success of such a therapy is determined by the implant's ability to interact positively with the host tissue (e.g., cell invasion, degradation and replacement). The second type of applications, i.e. fundamental research, focuses on understanding the fundamental aspects of bone biology and the assessment of drugs by developing in vitro models. This involves the development of scaffolds for the in vitro culture of cells in controlled three-dimensional (3D) environments. ${ }^{[3]}$

For both therapeutic and 3D-scaffold-based culture strategies, the scaffold architecture may directly (e.g., nutrient transport, cell colonization) or indirectly (e.g., shear stress, local stimuli) influence the cell fate through its macroscopic $(>100 \mu \mathrm{m})$ and microscopic $(<20 \mu \mathrm{m})$ features. Numerous studies have investigated the influence of micro- and macro- architecture, both of which are of great importance (see for review). ${ }^{[3]}$ However, an important disparity regarding the influence of the architecture on cell response and fate is present in the current literature as summarized in Bouet et al. ${ }^{[3]}$ Such discrepancies are most likely due to the random internal pore architecture of the scaffolds, which is related to the limitations of current scaffold production methods.

Until recently, CaP bioceramics have been primarily produced using traditional ceramic processing techniques. Depending on the intended applications, several approaches may be considered, including the use of CaP dry powders (e.g., pressing), ${ }^{[4]}$ bulk materials (e.g., hydrothermal exchange, machining) ${ }^{[5]}$ or suspensions. Suspension-based methods, such as porogen or salt leaching, ${ }^{[6]}$ mold impregnation, ${ }^{[7]}$ foaming, ${ }^{[8]}$ or freeze drying, ${ }^{[9]}$ are traditional and versatile processes for manufacturing of macroporous CaP scaffolds. ${ }^{[10]}$ These conventional methods are relevant and allow the production of tuned scaffolds; however, they still suffer from architectural limitations (e.g., global/local shape), internal inhomogeneity (e.g., pore and interconnection size) and sample-to- 
sample variations (e.g., random pore distribution). This lack of architectural standardization prevents the rigorous design of biological experiments and limits their reproducibility. ${ }^{[3]}$

New engineering developments that combine computational methods and additive manufacturing (AM) technologies are able to overcome such limitations by providing both a higher level of control over the design of the manufactured scaffolds and the opportunity to create reproducible, optimized custom architectures. AM technologies for bioceramics are far less advanced than for polymers and metals, ${ }^{[11]}$ but prototypes or small batches of $\mathrm{Al}_{2} \mathrm{O}_{3} / \mathrm{ZrO}_{2}$ have been produced using stereolithography (SLA, e.g., Lithoz, Austria; Prodways, France), selective laser melting (SLM, e.g., Phenix systems, France) or 3D-printing (3DP, e.g., Center for Fine Print Research, UK) technologies. Technological development of AM methods for the production of CaP scaffolds is still in its early stages because of the challenging specifications of biological applications associated with the specific physicochemical ${ }^{[12]}$ and thermal properties ${ }^{[13]}$ of CaP. Thus, the AM process for the production of such CaP scaffolds should preserve CaP biocompatibility (no toxic residue), should not induce phase modifications, and should allow reproducible and precise manufacturing of a wide array of custom architectures (e.g., pore sizes from approximately $150 \mu \mathrm{m}$ to $1 \mathrm{~mm}$ ). Additional criteria, such as the AM machine costs and the flexibility and ease of implementation of the process must also be considered.

CaP scaffolds produced via "direct-write assembly", ${ }^{[14]}$ including robocasting ${ }^{[15]}$ or 3DP, ${ }^{[16]}$ have been recently reported in the comprehensive reviews of Kumar et al. ${ }^{[17]}$ The conclusion is that their applications are restricted due to the lack of architectural control at both the global and local scales and the architectural limitations inherent in these processes (e.g., arrangement of tubes more or less well-defined for "3D plotting", ${ }^{[17 b]}$ accuracy of deposition and post-printing cleaning of the structures for 3DP). Craniofacial bone substitutes were successfully produced by SLA (3DCeram, France). ${ }^{[18]}$ Nonetheless, apart from the high costs of such a technology, the SLA process involves the use of toxic monomers, ${ }^{[19]}$ which may affect sensitive CaP phases, and lacks flexibility; especially because of the 
difficulty in adapting the compositions of the highly concentrated ceramic suspensions ${ }^{[20]}$ to various CaP phases. Finally, post-processing or post-fabrication issues, such as the removal of nonpolymerized slurry or, in some case, support structures, restrict the ceramic design possibilities. ${ }^{[17 b, 21]}$ Selective laser sintering (SLS) ${ }^{[22]}$ and SLM ${ }^{[23]}$ methods require further developments for the production of CaP scaffolds, primarily due to the low macro- and micro-architectural control and to phase degradation during the shaping process that is not compatible with the intended biological applications. Moreover, because these technologies use a laser beam or electron beam as the power source, they are expensive, and their use in research laboratories is limited.

Considering the specifications and current limitations (listed above) for the production of CaP bioceramics, we utilizes an "indirect 3D printing" as defined in Do et al., ${ }^{[24]}$ and more precisely a manufacturing process based on the impregnation of wax molds produced via an AM technology. This process already described in recent studies, ${ }^{[25]}$ was here optimized in order to fabricate pure CaP porous bioceramics with a high level of control over the architecture. More precisely, in this paper, we focus on the determination of the robustness, architectural capabilities and limitations of such a process to produce HA bioceramic. Wax molds were produced using a 3D Drop on Demand (DoD) based 3D printer, followed by a traditional ceramic process of replication. The optimization of the ceramic processing is described elsewhere. ${ }^{[26]}$ The influence of the wax mold printing strategy (e.g., orientation, resolution) on the micro- and macro- architectural features of the bioceramics was studied. Finally, the architectural reproducibility (e.g., shape conservation, sample-to-sample homogeneity) was assessed at the scaffold scale using triply periodic spherical structures with 8-mm diameters.

\section{Materials and Methods}

\subsection{Ceramic Manufacturing process - Overview.}

The manufacturing process consists of three main stages. 
The first stage is the computer-aided design (CAD) conception of the intended ceramic and its respective wax mold (Figure $1 \mathrm{~A}$ to $\mathrm{C}$ ). The latter is designed as the negative of the bioceramic (i.e., the structure and internal voids of the mold will become the macropores and the ceramic part of the scaffold, respectively). For compatibility purposes with the AM machine, CAD files are exported in the stereolithography file format (.stl), which is an approximation of the original model using planar triangles via tessellation. The material deposition (print head path) is determined using a virtual orientation of the mold .stl file that is sliced into a stack of 2-dimensional (2D) layers based on a given layer thickness. Finally, the sliced file is sent to the AM machine for the production stage.

The second stage is the production of the mold via additive manufacturing. A 3ZStudio 3D printer (Solidscape, Multistation, Paris) was selected because of its relatively low cost, high and adjustable resolution (from $6 \mu \mathrm{m}$ to $25 \mu \mathrm{m}$ ) and compatibility of wax molds with the ceramic processing (i.e., low shrinkage, adapted thermal properties and no residue after debinding). ${ }^{[26]}$ Molds were built layer by layer by depositing "build" and "support" molten wax droplets through outlet nozzles that harden immediately onto the piece under construction. The layer thickness is corrected using a milling head (Figure 1D). After the printing is completed, the part is placed in a $52^{\circ} \mathrm{C}$ bath of petroleum distillate (Onyx Ardéa, France) until the part is clean and all support material has been removed. Both the layer thickness and the mold printing orientation, shown in Figure $1 \mathrm{D}$ to $\mathrm{F}$, affect the mold features and, consequently, the resulting bioceramic. The effect of these parameters will be studied hereafter.

The ceramic processing, presented in section 2.2 and described in detail in Charbonnier et al., ${ }^{\text {[26] }}$ represents the final stage of the bioceramic production. Molds are impregnated with an aqueous suspension of CaP powder. After drying, the organic substances (e.g., wax mold, dispersing agent) are removed from the green material (debinding), which is then sintered to obtain the bioceramic.

The bioceramic manufacturing process, from mould manufacturing to ceramic sintering, induces large (i.e., at the scaffold scale) or localized morphological deformations, which may be detrimental 
to the bioceramic in terms of mechanical handling and/or compliance with specifications. Therefore the estimation of the morphological deviations of the produced bioceramics from their respective CAD models is required to validate this manufacturing process (see sections 2.3 and 2.4 ).

\subsection{Ceramic processing}

\subsubsection{Powder synthesis}

HA powder was prepared via an aqueous precipitation method using a fully automated apparatus. A diammonium hydrogen phosphate $\left(\left(\mathrm{NH}_{4}\right)_{2} \mathrm{HPO}_{4}, 99 \%\right.$, Merck, Germany) solution was added dropwise to a calcium nitrate solution $\left(\mathrm{Ca}\left(\mathrm{NO}_{3}\right)_{2} \cdot 4 \mathrm{H}_{2} \mathrm{O}, 99 \%\right.$, Merck, Germany) using peristaltic pumps. The reaction was performed under continuous stirring under reflux at a regulated temperature of $45^{\circ} \mathrm{C}$ (external T-probe). An argon flow (Air Products, $0.1 \mathrm{~L} / \mathrm{min}$ ) in the reactor prevented any uncontrolled carbonation of precipitates. The $\mathrm{pH}$ of the suspension was automatically adjusted and maintained at 9.0 with a $28 \%$ ammonia solution (Merck, Germany) by mean of a pH stat (GALa 0420 TTT, Prominent, Strasbourg, France). After the introduction of all of the reagents, the suspension was aged for $17 \mathrm{~h}$ and then filtered. The precipitate was pre-calcined at $400^{\circ} \mathrm{C}$ for 2 hours (Carbolite, UK) before it was crushed to a size of 1 to $10 \mu \mathrm{m}$ (Hosokawa Alpine, AFG100, Germany). The HA powder was then heat-treated at $1020^{\circ} \mathrm{C}$ for 2 hours (LH40/13, Nabertherm, Germany) to obtain a surface area of $4.0 \pm 0.1 \mathrm{~m}^{2} / \mathrm{g}(\mathrm{N}=3)$. This value was determined on powders outgassed at $150^{\circ} \mathrm{C}$ for $8 \mathrm{~h}$ by means of the Brunauer-Emmett-Teller (BET) 5 points method using the $\mathrm{N}_{2}$ adsorption isotherm (Micromeritics ASAP 2010, Germany).

\subsubsection{Bioceramic scaffold preparation}

Templates were impregnated with a HA slurry prepared by blending $73.6 \%(\mathrm{w} / \mathrm{w})$ powder, $26.2 \%$ (w/w) pure water and 0.2\% (w/w) dispersing agent (Darvan C-N,R. T. Vanderbilt Company Inc., USA) for $15 \mathrm{~min}$ at $200 \mathrm{rpm}$ in a zirconia jar with 10 and $5 \mathrm{~mm}$ diameter zirconia balls (PM400, Retsch, 
Germany). An organic binder (Duramax B-1000, Rohmand Haas, France) was then added at a concentration of $3.7 \%$ (w/w of slurry). The slurry was then mixed for 15 min using a propeller stirrer. After drying overnight at room temperature, the green bodies were cleaned of all excess dried slurry using a surgical blade (Swann-Morton, UK). The green bodies were then heat-treated in a debinding furnace (Carbolite, UK) at a temperature of $500^{\circ} \mathrm{C}$ to eliminate the polymer mold and the organic adjuvants. The consolidation of the ceramic was achieved by sintering at $1200^{\circ} \mathrm{C}$ for 2 hours in air with a heating rate of $4^{\circ} \mathrm{C} / \mathrm{min}$ (LH40/13, Nabertherm, Germany).

\subsection{Influence of mold printing parameters on ceramic architectural features}

A bioceramic CAD model with channels oriented along a single direction (Figure $1 \mathrm{~A}$ ) was designed to assess the influence of the mold printing parameters on the architectural features of the bioceramic. Circular and right-angled triangular $\left(\theta_{1}=90^{\circ}, \theta_{2}=45^{\circ}\right)$ channels were defined with three different sizes according to macropore sizes reported in the literature: ${ }^{[3]}$ small $(\approx 200 \mu \mathrm{m})$, medium $(\approx 400 \mu \mathrm{m})$ and large $(\approx 800 \mu \mathrm{m})$. The dimensions of the round and triangular columns of the molds printed with resolutions of $6 \mu \mathrm{m}, 12 \mu \mathrm{m}$ and $25 \mu \mathrm{m}$ along the z-axis (Figure 1E) or perpendicular to the printer $\mathrm{x}$ axis (Figure 1F) are presented in Figure 1. Bioceramics were obtained according to the process described in section 2.2 .

Morphometric analyzes of the produced bioceramics were carried out at various scales. Each bioceramic was first imaged using a Nanotom S X-ray computed tomography system (Phoenix, USA) with a voltage of $80 \mathrm{kV}$ (tungsten target), an integration time of $750 \mathrm{~ms}$ and a $3.5 \mu \mathrm{m}$ voxel resolution. For reconstruction of the volume data, a proprietary implementation based on the Feldkamps cone beam-reconstruction algorithm was used. VG Studio software (Volume Graphics, Heidelberg, Germany) was used for the 3D visualization of the volume data and the dataset export in .DICOM format for image analysis. The geometrical features of the bioceramic pores were analyzed in $2 \mathrm{D}$ on 3 cross-sections perpendicular to the channels at the inlet, middle and outlet of the 
bioceramic using ImageJ freeware (National Institutes of Health, USA). A schematic representation for right-angled triangular channel is given in Figure 2. From these results, three morphological factors, aspect ratio (A.R.), form factor (F.F.), and roundness (R.) were calculated from the following Equations and in accordance with ISO norm 9276-6:

A.R. $=\frac{B}{L}$

$F . F .=\sqrt{\frac{4 \times \pi \times A}{P^{2}}}$

$R .=\sqrt{\frac{4 \times A}{\pi \times L^{2}}}$

with $L$ and $B$ the maximum and minimum Feret diameters (see Figure 2), respectively, and $A$ and $P$ the area and the perimeter of the channel, respectively.

The theoretical values of the morphological factors A.R., F.F. and R. are equal to 1 for a circle, and $0.50,0.73$ and 0.56 for isosceles right triangle, respectively.

Scanning electron microscopy (SEM, JEOL JSM-6500F, USA) was used to image the internal geometry, microarchitecture and microstructure of the channels. Particular attention was given to the determination of the cusp depth $\left(\mathrm{H}_{\text {cusp }}\right)$ and spacing $\left(\mathrm{L}_{\text {cusp }}\right)$ inherent to the AM process (Figure 2$)$. Prior to observation, the bioceramics were mounted in a thermoset epoxy resin (EpoThin, Buehler, USA), with both of their faces covered to prevent the resin from entering the channels. After mounting, samples were polished either parallel or perpendicular to the channels, using an automated polishing system (Prepamatic, Struers, Denmark). Polished samples were coated with approximately $20 \mathrm{~nm}$ of gold (Q150R ES, Quorum Technologies) prior to imaging. 


\subsection{Architectural control and reliability at the scaffold scale - Process validation}

The targeted applications for the bioceramic scaffolds require reproducible production of complex 3D CaP scaffolds with a custom, controlled architecture. Consequently, the validation of our process requires an analysis of the architectural reliability at the scaffold scale. Spherical ceramics $(8 \mathrm{~mm}$ diameter) with a triply periodic internal architecture (gyroid), were designed using the ScanIP software suite (Simpleware, UK) and then manufactured to evaluate (i) the reproducibility of the process, (ii) the conservation of the shapes at both the global and local scales, and (iii) the homogeneity of the internal architecture and microstructure (Images of the CAD model are provided in supplementary Figure 1). This triply periodic porous network is comprised of $540 \mu \mathrm{m}$ diameter cavities with $330 \mu \mathrm{m}$ diameter interconnections and $310 \mu \mathrm{m}$ thick ceramic walls. An $850 \mu \mathrm{m}$ gyroid unit cell was repeated over the entire sphere volume, resulting in a total porosity of $70 \%$. A cylinder $(\varnothing=850 \mu \mathrm{m}, \mathrm{h}=850 \mu \mathrm{m})$ and a cube $(\mathrm{L}=850 \mu \mathrm{m})$ were inserted into the structure as visual markers for further geometric data analysis (see supplementary Figure 1). Molds were printed at a resolution of $6 \mu \mathrm{m}$ and the HA bioceramics were produced in triplicate according to the process described in section 2.2 .

Morphometric analysis was performed using X-ray tomography (see Section 2.3). ScanIP suite was used for image data processing. The conservation of the spherical shape of the bioceramics was first analysed. To assess the shape conservation of the internal architecture over the entire sphere volume, cross-sections of the $x y^{-}, x z-$, and $y z-$ planes were compared to the CAD file to evaluate any deviations. The CAD model and the bioceramic 3D images were superimposed using the square and cylindrical markers and a uniform global shrinkage of $14 \%$. 


\section{Results}

\subsection{Influence of the mold printing parameters on the scaffold architectural features}

Figure 3 shows $\mathrm{X}$-ray micro-tomography 3D images of the bioceramics and their respective cross sections obtained from molds printed with columns along (Figure 3A) or perpendicular (Figure 3B) to the z-axis of the printer (Figure 1). The printing orientation of the mold is visible on the ceramics printed at resolutions of $25 \mu \mathrm{m}$ (images not included) and $12 \mu \mathrm{m}$ (Figure 3), but is much less apparent for a printing resolution of $6 \mu \mathrm{m}$ (images not included). A more detailed examination of these observations is presented in Section 3.1.

At the macroscale, the bioceramics exhibit all 6 channels regardless of the manufacturing parameters. Despite cropping of the $90^{\circ}$ angles, triangular shaped channels are sharper and betterdefined in bioceramics with channels perpendicular to the z-axis of the printer (Figure 3B), than in bioceramics with channels parallel to the z-axis of the printer (Figure $3 \mathrm{~A})$. In the latter, the vertices of the triangular shapes are rounded, so much so that the smallest triangle appears circular, regardless of the mold printing resolution. Circular shapes are generally well defined; however, a clear deformation is observed on the smallest circle in Figure 3B which appears as a square.

These general observations are complemented by the analysis of the geometric characteristics and morphological factors displayed in Figures 4 and 5, respectively.

In bioceramics produced from molds with columns aligned along the $\mathrm{z}$-axis of the printer (Figures $3 \mathrm{~A}$ and $4 \mathrm{~A}$ ), the diameter of the circular channels (Figure 4A1) changes slightly from sample to sample, with a maximum deviation of $15 \mu \mathrm{m}$, but evolves with the resolution. For instance, the diameter of the smallest channel decreases from 232 to $192 \mu \mathrm{m}$ as the resolution increases from 25 to $6 \mu \mathrm{m}$. In contrast, when the mold is printed with columns perpendicular to the z-axis of the printer (Figures 3B and $4 \mathrm{~B}$ ), there is no significant variation in the bioceramics channel diameter regardless of the resolution. Thus, the diameters of small, medium and large circular channels are centered at 195, 
425 and $820 \mu \mathrm{m}$ with a maximum standard deviation of $10 \mu \mathrm{m}$. Conservation of angles in the triangular channels compared to the CAD model is highly dependent on the triangle size as well as the printing orientation of the molds. For bioceramics produced from molds with columns along the z-axis of the printer (Figure 4A2-A3), no angle was measured on the smallest triangle due to its circular shape, but the medium and large triangles exhibit relatively good angular conservation (e.g., $43.6^{\circ}, 45.0^{\circ}$ and $45.0^{\circ}$ for large triangles with a 25,12 and $6 \mu \mathrm{m}$ resolution). The standard deviation decreases with increased triangle size: non-measurable, $\pm 1.1, \pm 0.5^{\circ}$ for $45^{\circ}$ angles of small, medium and large triangles, respectively. Moreover, the angle variation, with regard to the theoretical values $\left(45^{\circ}\right.$ or $\left.90^{\circ}\right)$, narrows slightly with an increase in the printing resolution (e.g., $\pm 1.15^{\circ}> \pm 1.1^{\circ}> \pm 1.05^{\circ}$ for 25,12 and $6 \mu \mathrm{m}$ resolution, respectively, for medium triangle $45^{\circ}$ angles). These observations remain mostly valid for the bioceramics produced from molds with columns perpendicular to the zaxis of the printer, with two differences (Figure 4B2-B3). First, there is better conservation of the angles in the smallest triangle. Although the angles are $82 \pm 1.3^{\circ}$ and $48.5 \pm 0.9^{\circ}$ instead of $90^{\circ}$ and $45^{\circ}$ angles, respectively, we were able to measure these angles unlike in the bioceramics produced from molds with columns along the z-axis of the printer. Second, there is better angular control because the standard deviation and angular variation are lower for both the medium and the large triangles (i.e., $47.3 \pm 0.6^{\circ}, 45.4 \pm 0.6^{\circ}$ and $45.5 \pm 05^{\circ}$ for resolutions of 25,12 and $6 \mu \mathrm{m}$, respectively, for the $45^{\circ}$ angles in the medium triangle).

Aspect ratios, roundness, and form factors for the triangular and circular channels are displayed in Figure 5 for each set of manufacturing parameters.

For bioceramics manufactured from molds with columns both along and perpendicular to the z-axis of the printer, the aspect ratio is close to its theoretical value for the medium and large channels regardless of the shape (triangular or circular). A deviation from the theoretical value is observed for the small circular and triangular channels. This deviation slightly decreases with increased printing resolution. Channel roundness follows a similar trend with the exception of the triangular channels of 
the bioceramics produced from molds printed with columns perpendicular to the z-axis of the printer, which have roundness values that match the theoretical values regardless of channel size. Finally, the form factor matches or is close to its theoretical value regardless of the shape of the channels and the printing orientation of the mold. An exception to this trend can be observed for the circular channels of the bioceramics produced from molds with columns perpendicular to the z-axis of the printer. Finally, when molds are printed with columns perpendicular to the z-axis, the form factors of the bioceramics channels approach the theoretical values as the resolution increases.

Figure 6 shows SEM images of cross-sections of the circular channels for both mold printing directions. The images show the layer-by-layer structure of the bioceramic induced by the mold topology.

The $25 \mu \mathrm{m}$ layer resolution resulted in a surface topology on the produced bioceramic with $\mathrm{H}_{\text {cusp }}=$ $19.8 \pm 3.0 \mu \mathrm{m}$ and $\mathrm{L}_{\text {cusp }}=20.7 \pm 1.9 \mu \mathrm{m}$. Likewise, using a $12 \mu \mathrm{m} 3 \mathrm{D}$ printer resolution, the bioceramics exhibit a surface topology with $\mathrm{H}_{\text {cusp }}=9.6 \pm 1.0 \mu \mathrm{m}$ and $\mathrm{L}_{\text {cusp }}=9.2 \pm 1.9 \mu \mathrm{m}$. For a $6 \mu \mathrm{m}$ resolution, the layer-by-layer and well-defined micro-architecture tend to blur, with cusps approximately $5 \mu \mathrm{m}$ wide and high, which sometimes merge to form broader grooves (Figure 6C). Furthermore, as the resolution increases, the channel surfaces become less jagged-looking and are more linear (images not included). Similar conclusions can be drawn for the bioceramics with channels perpendicular to the z-axis of the printer (Figure $6 \mathrm{D}$ to $\mathrm{F}$ ). The staircase morphology, observed for a $25 \mu \mathrm{m}$ resolution, becomes smoother as the resolution increases.

\subsection{Process robustness at the scaffold scale}

Figure 7A displays an X-ray micro-tomography 3D image of the triply periodic bioceramic. The spherical shape is preserved, with an apparent shrinkage of $14.5 \pm 0.8 \%$. At a local scale, the internal 
architecture is also maintained and does not undergo any inhomogeneous deformation with a wall shrinkage of $14.1 \pm 2.7 \%$ (Figure 7B).

The similarities between the bioceramic CAD model and the produced bioceramic are assessed (Figure 7C). Despite a lack of overlapping areas, especially at the edge of the ceramic, the gyroid shape is maintained over the entire sphere volume. The lack of overlapping areas may be explained by (i) the removal of excess material during the cleaning step of greens (see Section 2.2.2), and (ii) software limitations for 3D image fitting (e.g., maximal angular accuracy of $1^{\circ}$ ).

\section{Discussion}

In this work, we validated the manufacturing of CaP bioceramics with complex and controlled architectures via the impregnation of 3D-printed wax molds. The macroporous 3D networks are reproducible with a large amount of control at both the global and local scales (Section 3.2). Despite a high consistency between the produced 3D bioceramics and their respective CAD models, deviations in the construct surface may be observed. This was confirmed by the geometrical analysis performed at the pore scale (section 3.1). The shapes of the unidirectional channels were generally well-defined and the highest geometrical accuracy (with respect to angle) and dimensional reliability were achieved in bioceramics produced using molds with columns perpendicular to the z-axis of the printer. An increase in the layer resolution from 25 to $6 \mu \mathrm{m}$ significantly reduced the channel roughness (Figure 6). Nonetheless, shape deformations were not completely avoided (e.g., rounded edges or cropping of the $90^{\circ}$ angle of the triangles, see Section 3.1).

Some possible causes were identified to explain bioceramics architectural deviations from the CAD model: (i) issues during ceramic processing, (ii) inappropriate mold printing strategy, (iii) approximations in the .stl export of the mold CAD model or inaccuracy in the .stl file slicing, and (iv) technological limitations of the 3D printer. 
Ceramic processing (i.e., mold cleaning, impregnation and heat treatments) is not responsible for the differences between the bioceramic and its CAD model because the slurry formulation used herein was proved to be suitable for the developed process without generating post-sintering defects (i.e., homogeneous and isotropic shrinkage). ${ }^{[26]}$ Furthermore, complementary X-ray micro-tomography scans of the molds revealed similar deformation at both the mold and bioceramic surfaces. Finally, no wax residue or dust was observed in the mold, suggesting that the defects observed on the bioceramic surface are related to mold topology. This is supported by the fact that the shape deformations are specific to the mold printing parameters, and primarily a function of the mold printing orientation. In fact, the mold printing resolution affects the surface morphology more than the geometrical features (i.e., shapes, see Figure 2). In addition, for a given printing orientation, the architectural features are affected to different extents depending on their geometry (e.g., angles versus curves) and dimensions (e.g., small versus large channels). These trends are consistent with the technological challenges of 3D-printing based technologies as stated by Oropallo et al. ${ }^{[19 b]}$ The choice of a printing orientation appears to be a compromise between part design and one or more manufacturing considerations such as printing time, cost or geometrical accuracy. ${ }^{[27]}$ Thus, the more specifications required for a part, the more compromises required. It is also crucial to note that depending on the part design, "there may not be an optimal orientation that meets the needs of the design". ${ }^{[19 b]}$ This need for a printing strategy emphasizes the current limitations of AM processes, including computational methods (e.g., CAD file, tessellation).

It is generally accepted that the tessellation algorithms used for the export of the CAD model in .stl file can generate geometric errors, especially if the model contains curved surfaces. ${ }^{[28]}$ In our case, this has a minor impact as no facet or chordal error was observed on either the ceramics or the molds. Additionally, the .stl file slicing can lead to architectural deviations between the bioceramic and its CAD model. For columns printed along the z-axis of the printer, the CAD model is maintained after slicing (i.e., triangles keep sharp angles without deformation regardless of their size and circles undergo minor polygonal approximations). In the worst-case scenario, that of the smallest circle, this 
approximation produces an 18-sided polygon (side length of approximately $36.8 \mu \mathrm{m}$ ) with a mean diameter of $209.4 \pm 0.5 \mu \mathrm{m}$ rather than $220 \mu \mathrm{m}$ (theoretical value). Conversely, for an orientation of columns perpendicular to the z-axis of the printer, the .stl file slicing results in cropping of the $90^{\circ}$ angles, lightly flattened surfaces at the circle extremities (top and bottom) and staircase morphologies at the construct surfaces. Similar deformations are observed on the molds and the bioceramics. An example of the imperfections is illustrated in Table 1 with a comparison, at different production stages, of the flattened surfaces on the flat-topped triangles. For more precision, please refer to the supplementary Figure 2.

This cross-analysis (Table 1) clearly indicates that the slicing procedure is not the most significant cause of geometrical deviation from the CAD models. Even though the flattened surfaces measured on sliced and micro-tomography images are of the same order of magnitude (hundred micrometers), the flattened surfaces of the molds are, on average, $39.4 \pm 11.9 \%$ wider than in the sliced .stl files. This suggests that the DoD technology is the predominant limiting factor.

As presented briefly in section 2.1 , the mold is fabricated layer by layer, alternating between waxes deposition through outlet nozzles and correction of the layer thickness with a milling head. No significant shape deformation based on the milling direction was detected on the molds or the bioceramics. Thus, the technological limitations of the 3D printer are due to the wax deposition, more specifically to the variation of droplet size $(\Delta d)$, droplet position $(\Delta p)$, and droplet spreading on the material ( $\Delta \mathrm{s}$ average diameter of the splashed droplet after deposition) as presented in Figure 8. These variations are a function of the frequency of the piezo-electric component $(\Delta f)$ inside the printhead, the print-head and operating temperatures $(\Delta T)$, and the print head position and momentum. ${ }^{[29]}$ All these technical wax deposition issues result in variations of $\pm 17.1 \%$ in the deposit width (see Figure 8) for a single straight line monolayer ( $2.5 \mathrm{~mm}$ length, $\mathrm{N}=30$ ). This is consistent with the inaccuracies observed in the molds with unidirectional columns and their resulting bioceramics, independent of the printing parameters. 
Overcoming the current technological limits of the 3D printer through better control of the droplet size and deposition would improve this ceramic manufacturing method. Optimization of the proprietary slicing and print head path planning algorithms may also be required. Finally, the process limitations and possible printing strategies must be integrated into the ceramic design to allow compromises that are compatible with the intended applications. Currently, part design options are constrained by technological limitations but in the future, AM technologies may support better part design and lead to new scientific insight. However, for the current applications, the developed manufacturing process is far more accurate than conventional or current AM (e.g., direct-write assembly, SLS) ceramic processing methods and convenient enough for the production of custom bioceramics adapted to fundamental and therapeutic applications. Figure 9 illustrates the potential of our process from the control of the bioceramic design/shape up to the adjustment of the microand nano-features.

\section{Conclusions}

The goal of this study was to evaluate the reliability, robustness and limitations of a bioceramic manufacturing process based on the impregnation of additively manufactured molds. The results suggest that the architectural deviations observed between the CAD model and the bioceramic are primarily due to the technical limitations of the 3D printer, more specifically to the variability of the wax droplet deposition. However, the limits of this AM process are minor compared to the architectural opportunities it offers, including standardization of bioceramics manufacturing, high and reproducible architectural control for a wide range of designs, phase biocompatibility preservation and ease of implementation for various phase compositions. All of these advantages will allow the development of new tools for experimental/fundamental research that will help to better understand the influence of macro- and micro- architecture on cell response and fate. 


\section{Acknowledgements}

We thank Mr Georges Taillandier for his advices about the additive manufacturing machines and the "Region Rhône-Alpes" for the financial support provided for this work. 


\section{References}

[1] J. Lowry, Annals of The Royal College of Surgeons of England 2006, 88, 334.

[2] H. M. da Silva, M. Mateescu, C. Damia, E. Champion, G. Soares, K. Anselme, Colloids and Surfaces B: Biointerfaces 2010, 80, 138.

[3] G. Bouet, D. Marchat, M. Cruel, L. Malaval, L. Vico, Tissue Engineering Part B: Reviews 2014, 21, 133.

[4] R. Halouani, D. Bernache-Assolant, E. Champion, A. Ababou, Journal of Materials Science: Materials in Medicine 1994, 5, 563.

[5] J. Rocha, A. Lemos, S. Agathopoulos, P. Valério, S. Kannan, F. Oktar, J. Ferreira, Bone 2005, 37, 850.

[6] a) S.-S. Kim, M. Sun Park, O. Jeon, C. Yong Choi, B.-S. Kim, Biomaterials 2006, 27, 1399; b) X. Liu, L. A. Smith, J. Hu, P. X. Ma, Biomaterials 2009, 30, 2252.

[7] a) M. Descamps, T. Duhoo, F. Monchau, J. Lu, P. Hardouin, Hornez, A. Leriche, Journal of the European Ceramic Society 2008, 28, 149; b) M. Descamps, O. Richart, P. Hardouin, J. C. Hornez, A. Leriche, Ceramics International 2008, 34, 1131.

[8] A. Salerno, D. Guarnieri, M. lannone, S. Zeppetelli, P. A. Netti, Tissue Engineering Part A 2010, 16, 2661.

[9] S. Farhangdoust, A. Zamanian, M. Yasaei, M. Khorami, Materials Science and Engineering: C 2013, $33,453$.

[10] J. H. Shepherd, S. M. Best, JOM 2011, 63, 83.

[11] a) F. P. W. Melchels, Preparation of advanced porous structures by stereolithography for application in tissue engineering, University of Twente, 2010; b) J. P. Kruth, G. Levy, F. Klocke, T. H. C. Childs, CIRP Annals - Manufacturing Technology 2007, 56, 730.

[12] E. Boanini, M. Gazzano, A. Bigi, Acta Biomaterialia 2010, 6, 1882.

[13] E. Champion, Acta Biomaterialia 2013, 9, 5855. 
[14] J. A. Lewis, J. E. Smay, J. Stuecker, J. Cesarano, Journal of the American Ceramic Society 2006, 89, 3599.

[15] a) P. Miranda, A. Pajares, E. Saiz, A. P. Tomsia, F. Guiberteau, Journal of Biomedical Materials Research Part A 2008, 85, 218; b) J. G. Dellinger, J. Cesarano, R. D. Jamison, Journal of Biomedical Materials Research Part A 2007, 82, 383.

[16] a) A. Butscher, M. Bohner, S. Hofmann, L. Gauckler, R. Müller, Acta Biomaterialia 2011, 7, 907; b)

M. Castilho, C. Moseke, A. Ewald, U. Gbureck, J. Groll, I. Pires, J. Teßmar, E. Vorndran, Biofabrication $2014,6,015006$.

[17] a) A. Kumar, A. R. Akkineni, B. Basu, M. Gelinsky, Journal of biomaterials applications 2015, 0885328215617058; b) A. Kumar, S. Mandal, S. Barui, R. Vasireddi, U. Gbureck, M. Gelinsky, B. Basu, Materials Science and Engineering: R: Reports 2016, 103, 1.

[18] a) C. Chaput, T. Chartier, J. Brie, J. Lafon, Proceedings of the 4th International Congress on Ceramics 2012; b) J. Brie, T. Chartier, C. Chaput, C. Delage, B. Pradeau, F. Caire, M.-P. Boncoeur, J.-J. Moreau, Journal of Cranio-Maxillofacial Surgery 2013, 41, 403.

[19] a) V. K. Popov, A. V. Evseev, A. L. Ivanov, V. V. Roginski, A. I. Volozhin, S. M. Howdle, Journal of Materials Science: Materials in Medicine 2004, 15, 123; b) W. Oropallo, L. A. Piegl, Engineering with Computers 2015, 1.

[20] T. Chartier, C. Chaput, Y. ABOULLATIM, C. Delage, CFI. Ceramic forum international 2006, 83.

[21] G. Brunello, S. Sivolella, R. Meneghello, L. Ferroni, C. Gardin, A. Piattelli, B. Zavan, E. Bressan, Biotechnology advances 2016.

[22] a) B. Ma, L. Lin, X. Huang, Q. Hu, M. Fang, in Knowledge Enterprise: Intelligent Strategies in Product Design, Manufacturing, and Management, Springer, 2006, 710; b) C. Shuai, C. Gao, Y. Nie, H. Hu, Y. Zhou, S. Peng, Nanotechnology 2011, 22, 285703; c) C. Shuai, J. Zhuang, H. Hu, S. Peng, D. Liu, J. Liu, Biotechnology and applied biochemistry 2013, 60, 266; d) C. Shuai, P. Li, J. Liu, S. Peng, Materials Characterization 2013, 77, 23.

[23] C. Colin, J.-d. Bartout, E. Shaker, D. Marchat, D. Nimal, US Patent 20,160,052,162, 2016. 
[24] A. V. Do, B. Khorsand, S. M. Geary, A. K. Salem, Advanced healthcare materials 2015, 4, 1742.

[25] a) C. M. Bidan, K. P. Kommareddy, M. Rumpler, P. Kollmannsberger, Y. J. M. Bréchet, P. Fratzl, J. W. C. Dunlop, PLoS ONE 2012, 7, e36336; b) M. Rumpler, A. Woesz, J. W. C. Dunlop, J. T. van Dongen, P. Fratzl, J R Soc Interface 2008, 5, 1173; c) C. E. Wilson, J. D. de Bruijn, C. A. van Blitterswijk, A. J. Verbout, W. J. A. Dhert, J Biomed Mater Res A 2004, 68, 123; d) M. Schumacher, F. Uhl, R. Detsch, U. Deisinger, G. Ziegler, J Mater Sci Mater Med 2010, 21, 3039; e) J. Malmström, E. Adolfsson, L. Emanuelsson, P. Thomsen, Journal of Materials Science: Materials in Medicine 2008, 19, 2983.

[26] B. Charbonnier, C. Laurent, D. Marchat, Journal of the European Ceramic Society 2016.

[27] P. Pandey, N. V. Reddy, S. Dhande, Journal of materials processing technology 2007, 185, 125.

[28] G. Navangul, R. Paul, S. Anand, Journal of Manufacturing Science and Engineering 2013, 135, 031006

[29] I. Gibson, D. Rosen, B. Stucker, Additive manufacturing technologies: rapid prototyping to direct digital manufacturing, Springer, 2010. 
A

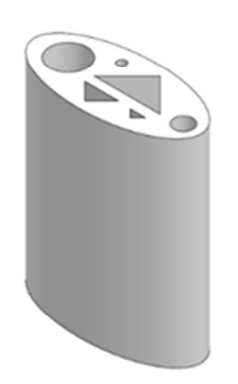

B

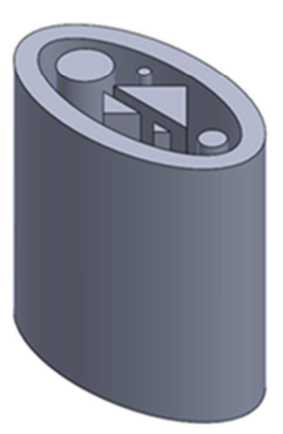

C

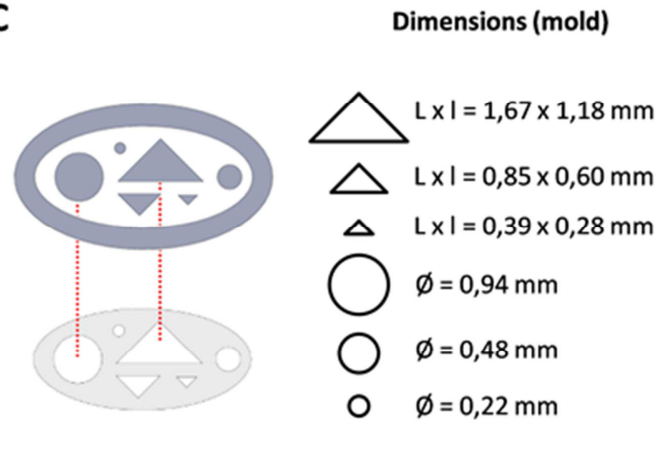
Dimensions (mold)

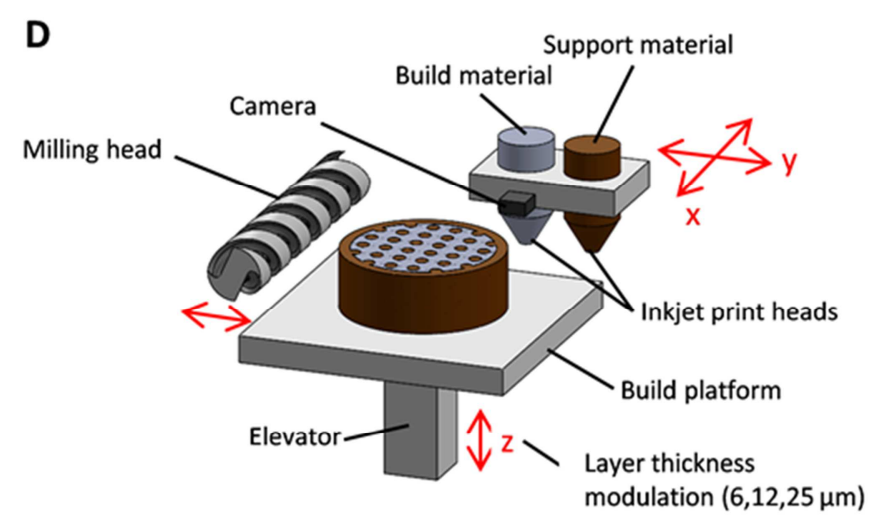

$\mathrm{E}$
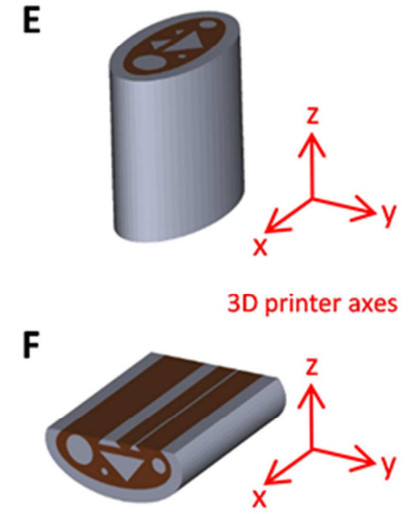

Figure 1. A) Ceramic and B) mold $3 D$ images derived from $C A D$ software, and C) their respective crosssections (Bioceramic channels are the negative of the mold columns, and triangles were designed with angles $\vartheta 1=45^{\circ}$ and $\vartheta 2=90^{\circ}$. Schematic representation of $D$ ) the $3 D$ printing technology with an adjustable layer thickness along the z-axis of the printer $(6,12,19,25 \mu \mathrm{m})$, and the printing orientation of molds with round and triangular columns E) along the z-axis and F) perpendicular to the z-axis 


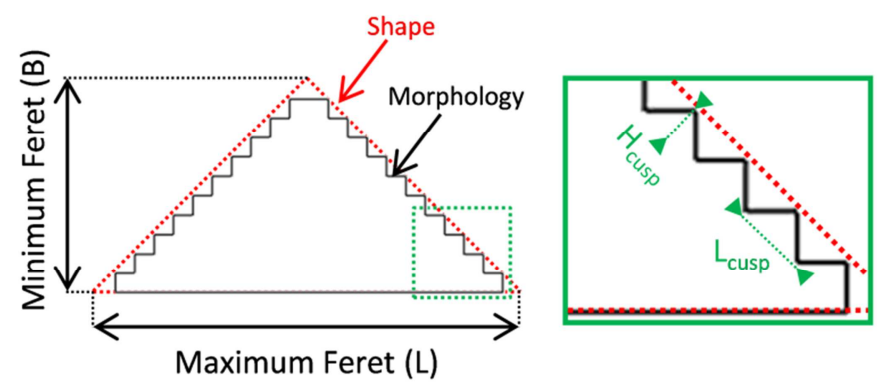

Figure 2. Schematic representation of the "shape" and "morphology" of an object, and the main dimensional variables considered for the geometric analysis at the pore scale.

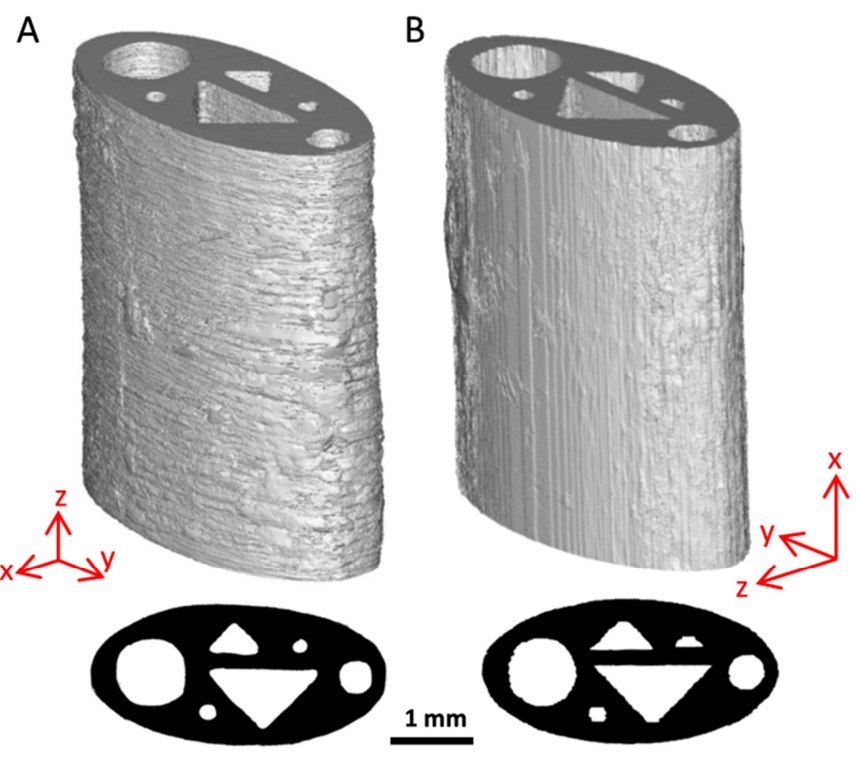

Figure 3. 3D X-ray micro-tomography images of bioceramics manufactured from molds printed with a $12 \mu \mathrm{m}$ resolution; the $x-, y-, z$ - axes represent the $3 D$ printer axis. Bioceramic from mold printed with columns A) along the z-axis and B) perpendicular to the z-axis of the printer. 


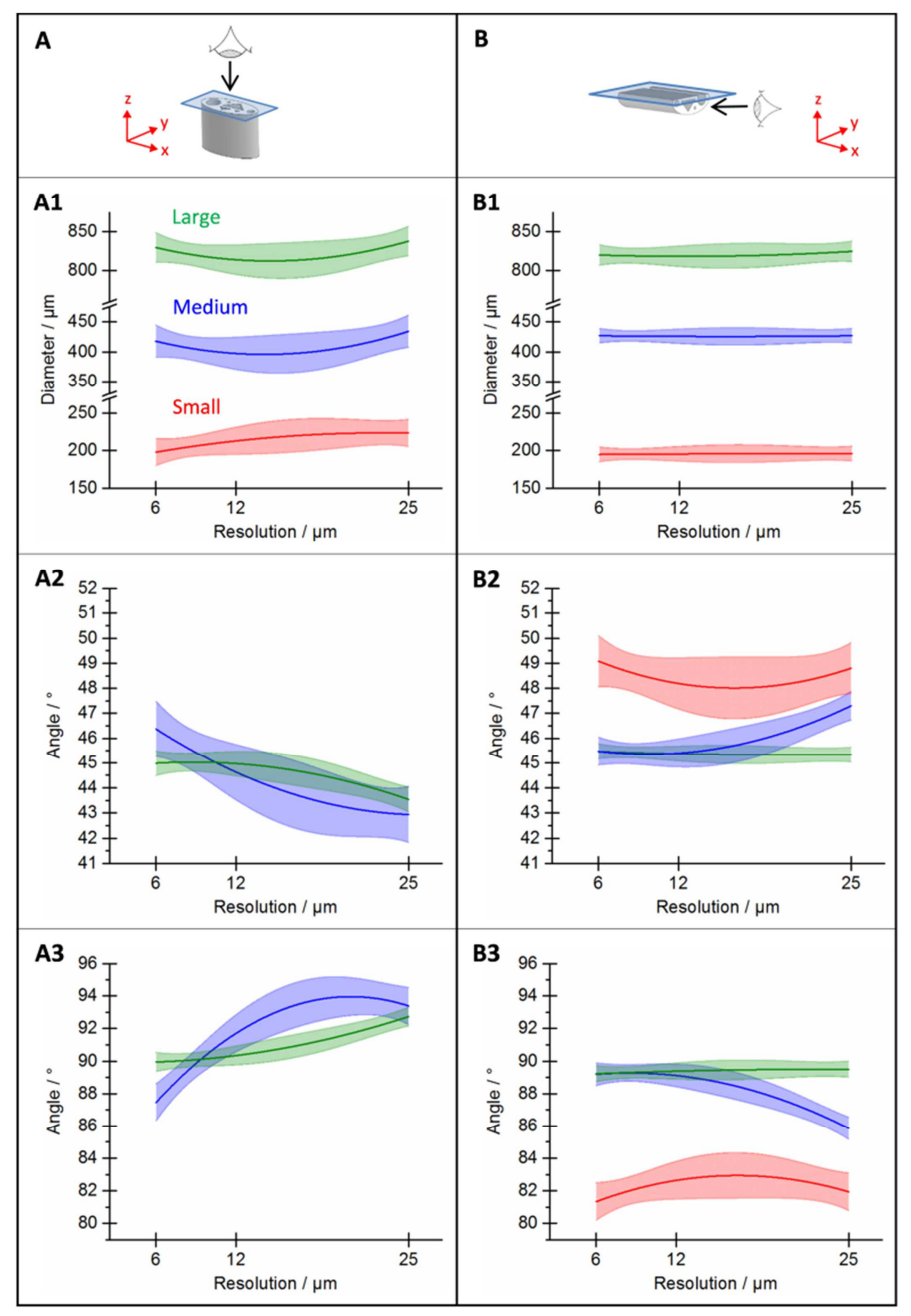

Figure 4. Influence of the mold printing strategy on the architectural features of the bioceramic manufactured from molds printed with columns A- along and B-perpendicular to the $z$-axis. The $x-, y$-, and z-axes represent the $3 D$ printer axes. $A 1-A 3$ and $B 1-B 3$ show the measured circles diameters and triangles $45^{\circ}$ and $90^{\circ}$ angles, respectively. Small, medium and large channels are displayed in red, blue and green, respectively. Colored bands outline the $99 \%$ confidence interval calculated from measurements $(N=9)$ with $2^{\text {nd }}$ degree polynomial fitted curves (OriginPro, OriginLab, USA). 


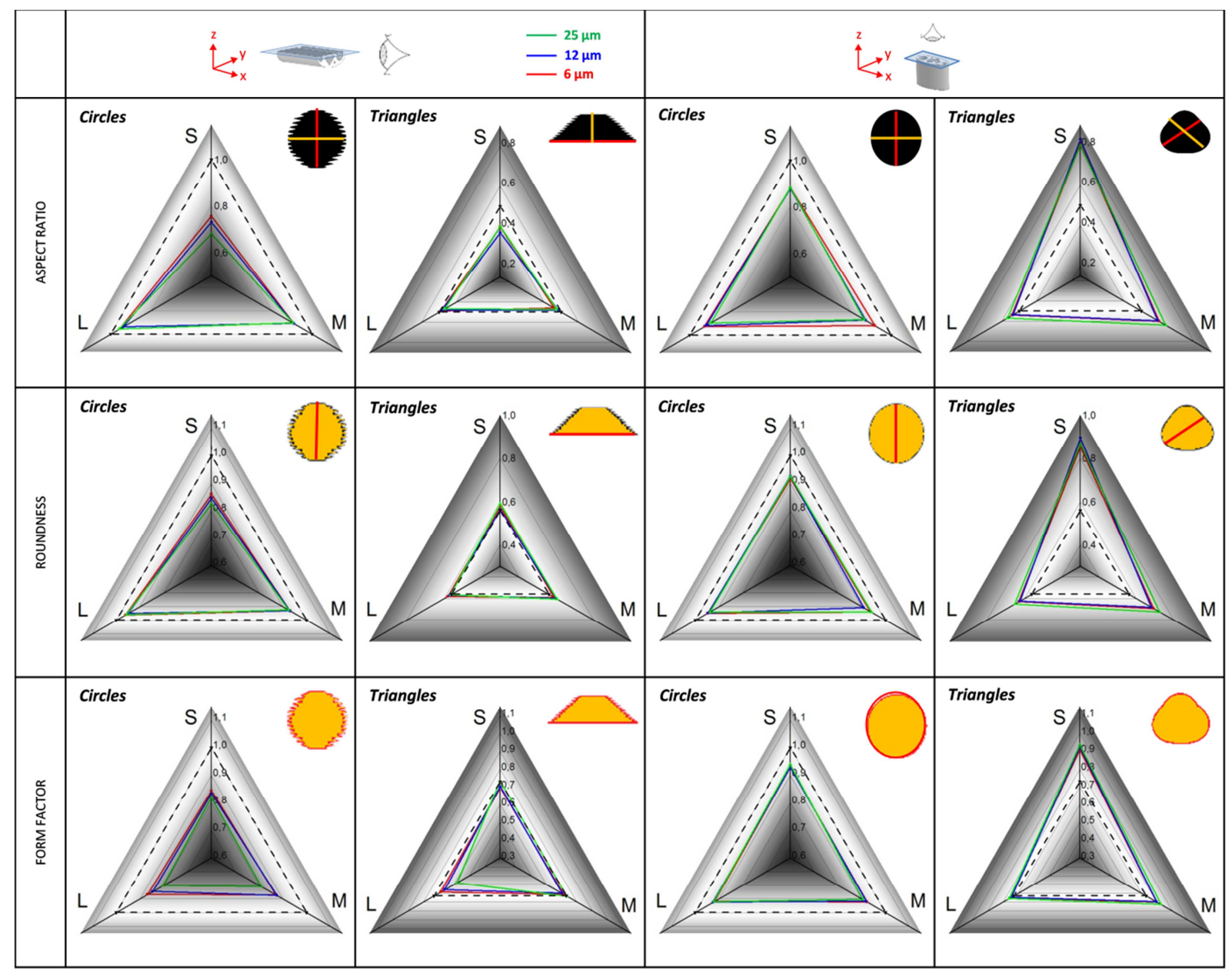

Figure 5. Influence of mold printing parameters on shape factors. The $S, M$ and $L$ symbols refer to the small, medium and large channels, respectively. Green, red and blue curves refer to the 25, 12 and 6 $\mu m$ resolution used to produce the molds, respectively. The $x-, y$-, and $z$-axes represent the printer axes. An overview of the general channel shape and parameters considered for the shape factor calculation (in orange and red) is displayed in the top, right corner of each cell. 


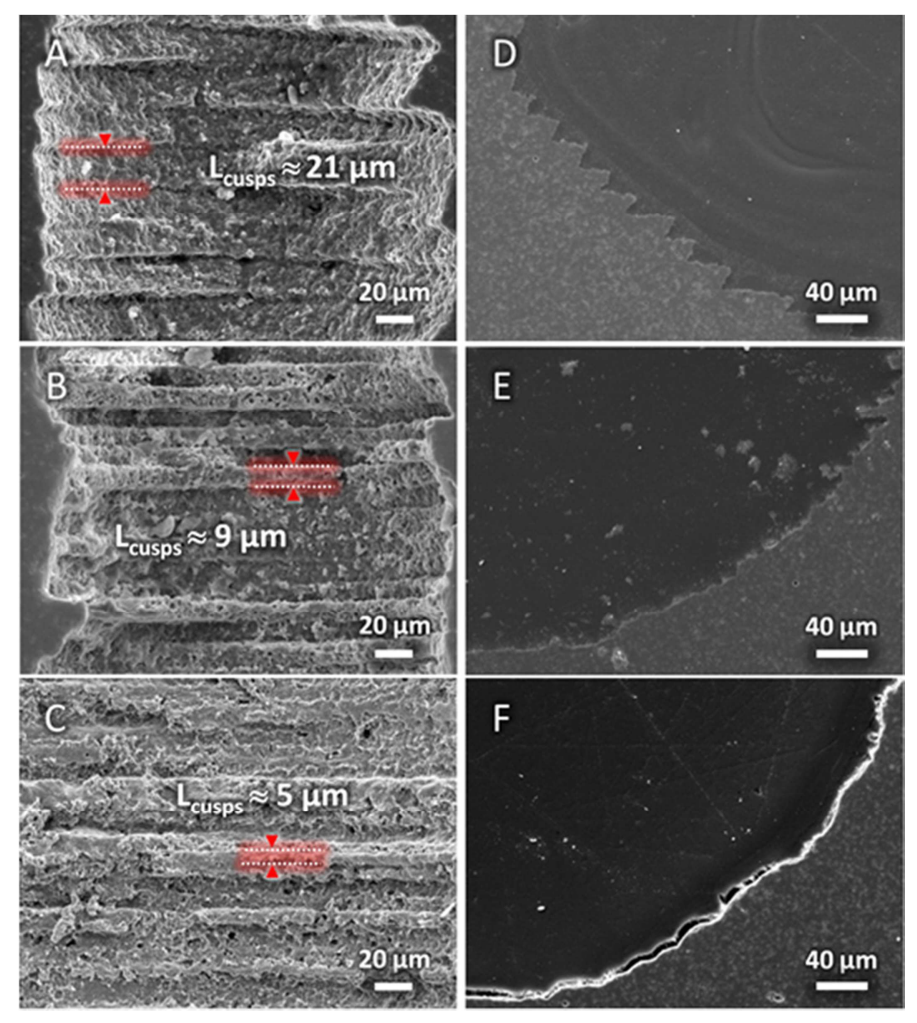

Figure 6. SEM images of the cross-sections of the channels in the bioceramics produced from molds printed with columns $A, B$ and C) along and $D, E$ and F) perpendicular to the z-axis of the printer with a 3D printer resolution of $25 \mu \mathrm{m}(A$ and $D), 12 \mu \mathrm{m}$ (B and $E$ ) and $6 \mu \mathrm{m}$ ( $C$ and $F$ ). 


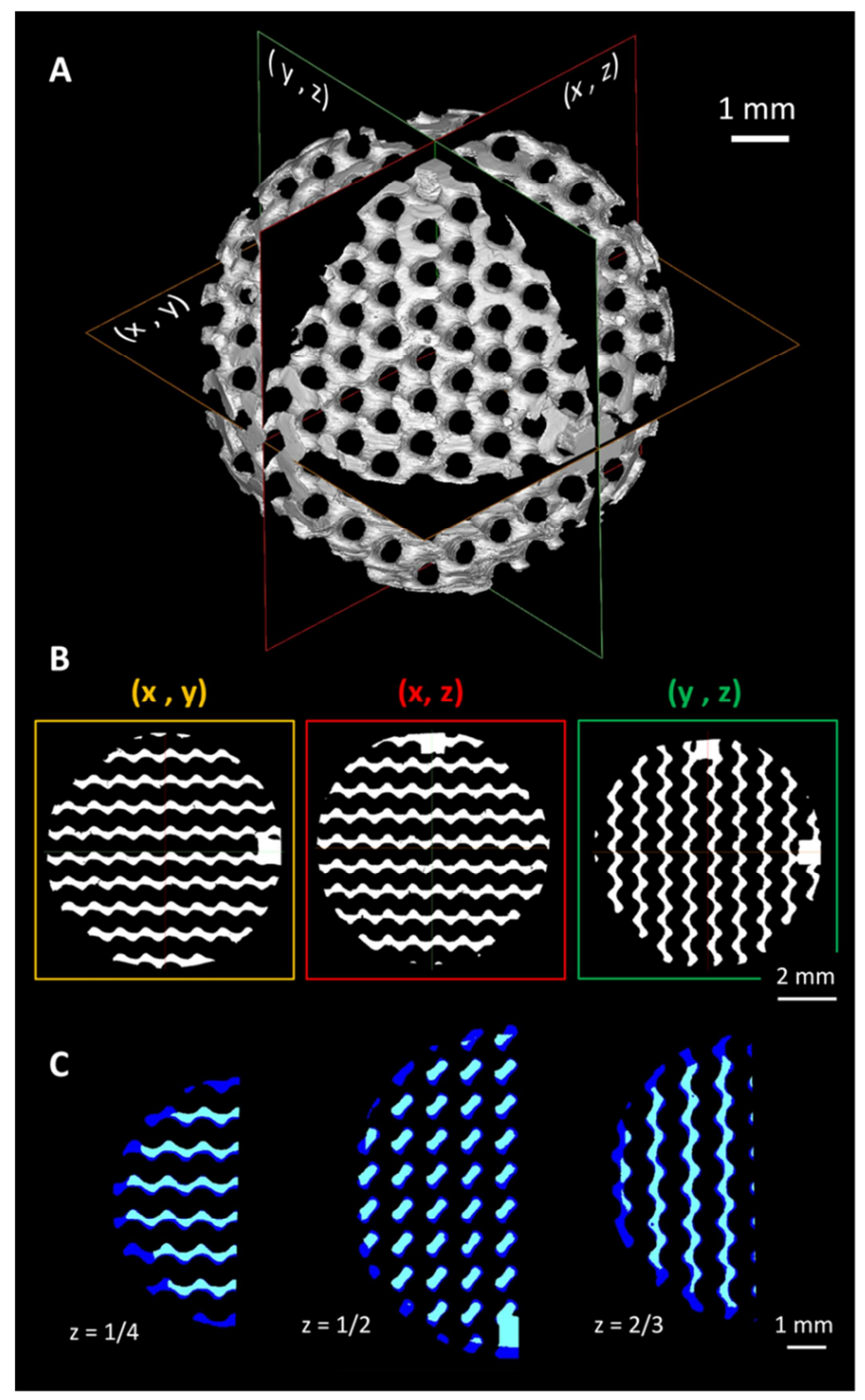

Figure 7. A) $3 D$ image obtained from X-ray micro-tomography of the spherical macroporous bioceramics (gyroid internal architecture), B) cross-sections relative to the $x y-, x z$ - and yz-planes displayed on the 3D view, and C) comparison of cross-sections of the CAD model (blue) and the resulting bioceramic (cyan) at different heights $(1 / 4,1 / 2,2 / 3)$ in the sample using Simpleware software (The shrinkage of the bioceramic structure was simulated on the CAD file using a homogeneous size reduction of 14\%). 


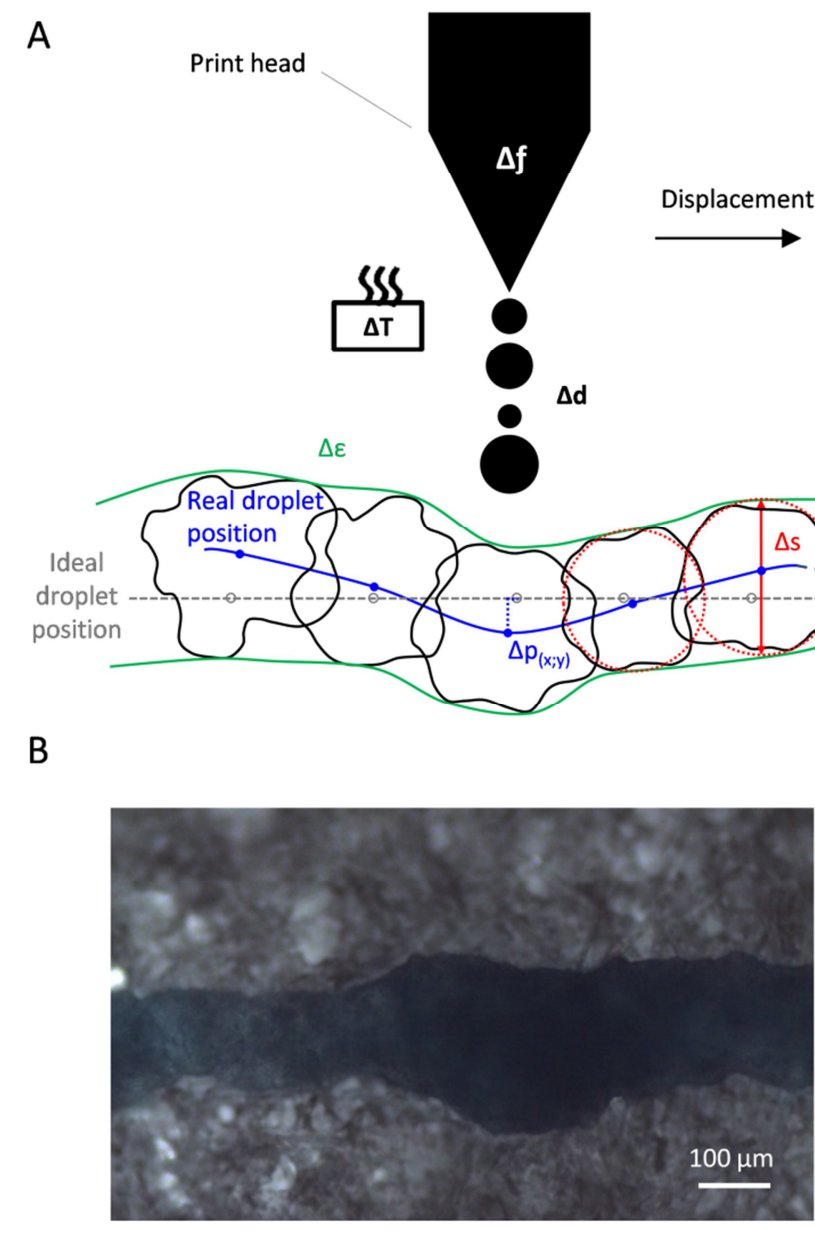

Figure 8. A) Schematic representation of the technological limitations of the DoD process, where $\Delta d$ is the size variation of the droplet size at the print head outlet, $\Delta p$ is the deviation of the wax droplet from its theoretical position, and $\Delta s$ is the average diameter of the splashed wax droplet after deposition. These wax deposition issues are greatly influenced by the frequency of the piezo-electric component $\Delta f$ inside the print-head, the print head and operating temperatures, $\Delta T$, as well as the print head position and momentum. B) Optical microscopy image of the resulting inaccuracy, $\Delta \varepsilon=$ $17.1 \%$, in the deposited material for a single straight line monolayer. 


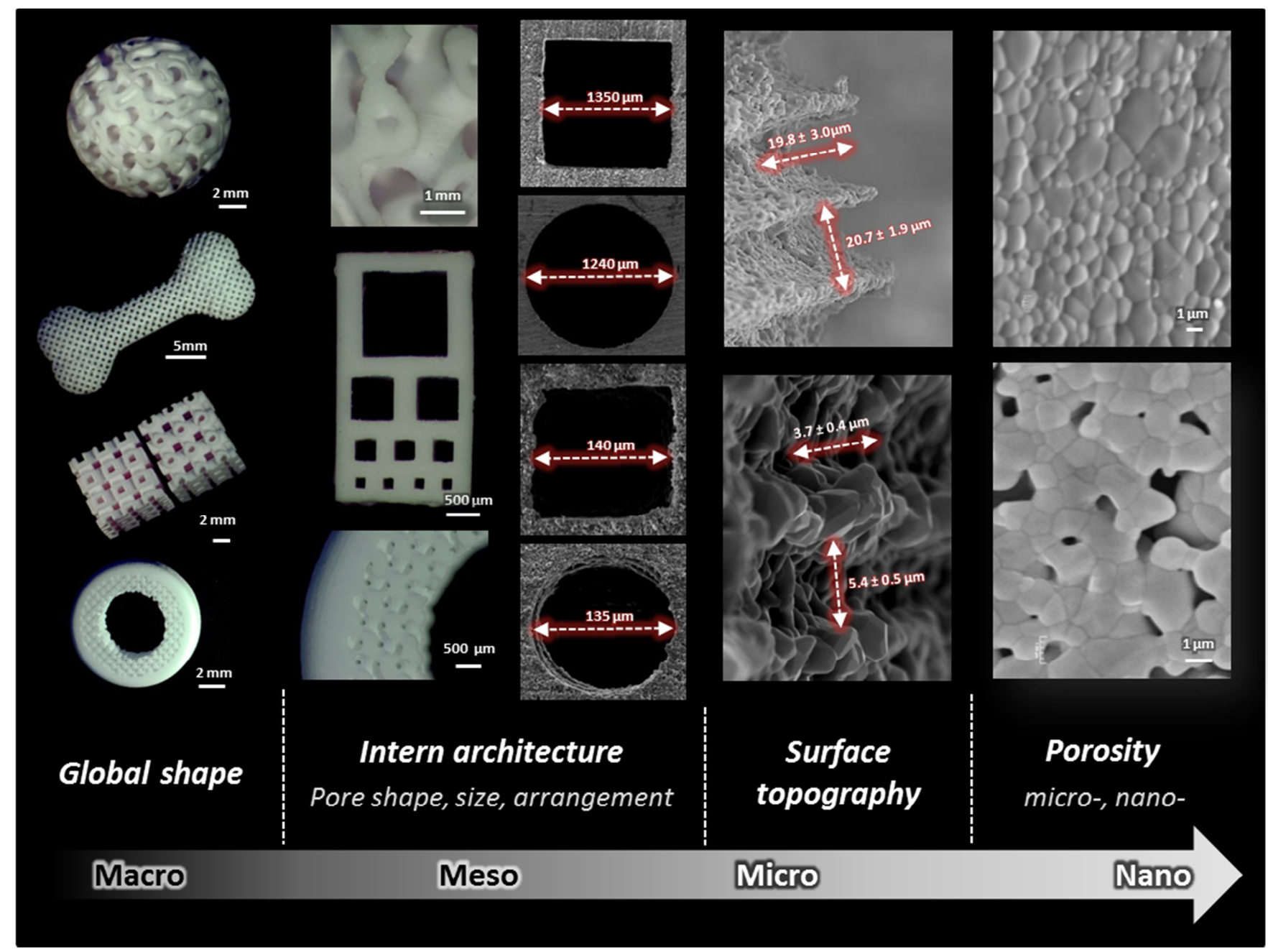

Figure 9. Examples of porous HA bioceramics produced by impregnation of 3D-printed wax molds.

This process allows adjusting the shape, the intern architecture (smallest achievable frame/shape (i.e., circle or square) is $120 \mu \mathrm{m}$ ), the surface microtopography (cusp depth and width of $20 \mu \mathrm{m}$ to 5 $\mu m)$ and the micro- nano-pores density of the pure bioceramics. 
Table 1. Lengths of the flattened surfaces derived from the cropping of the $90^{\circ}$ angle in the triangles.

Measurements $(N=9)$ were carried out on the sliced stl files and on X-ray micro-tomography images of the molds and bioceramics (orientation of the columns/ channels perpendicular to the z-axis of the 3D-printer). Fig. in the supplementary data complements this table.

\begin{tabular}{|c|c|c|c|c|}
\hline \multirow{2}{*}{ Resolution $/ \mu \mathrm{m}$} & \multirow{2}{*}{ Shapes } & \multicolumn{3}{|c|}{ Flattened surface / $\mu \mathrm{m}$} \\
\cline { 3 - 5 } & & Sliced .stl file & Mold & Bioceramic \\
\hline \multirow{3}{*}{25} & S Triangle & 109.9 & $155.0 \pm 4.0$ & $136.0 \pm 21.6$ \\
\cline { 2 - 5 } & M Triangle & 104.0 & $159.0 \pm 13.1$ & $146.7 \pm 13.6$ \\
\cline { 2 - 5 } & L Triangle & 116.2 & $180.3 \pm 3.1$ & $153.7 \pm 9.3$ \\
\hline \multirow{3}{*}{12} & S Triangle & 110.4 & $139.0 \pm 9.6$ & $133.7 \pm 10.6$ \\
\cline { 2 - 5 } & M Triangle & 103.0 & $134.0 \pm 11.5$ & $121.3 \pm 14.5$ \\
\cline { 2 - 5 } & L Triangle & 116.8 & $170.3 \pm 1.5$ & $147.3 \pm 12.6$ \\
\hline \multirow{3}{*}{6} & S Triangle & 109.7 & $129.0 \pm 5.3$ & $116.3 \pm 5.7$ \\
\cline { 2 - 5 } & M Triangle & 91.8 & $135.0 \pm 2.0$ & $128.3 \pm 2.3$ \\
\cline { 2 - 5 } & L Triangle & 116.7 & $162.3 \pm 3.5$ & $156.3 \pm 8.1$ \\
\hline
\end{tabular}

A

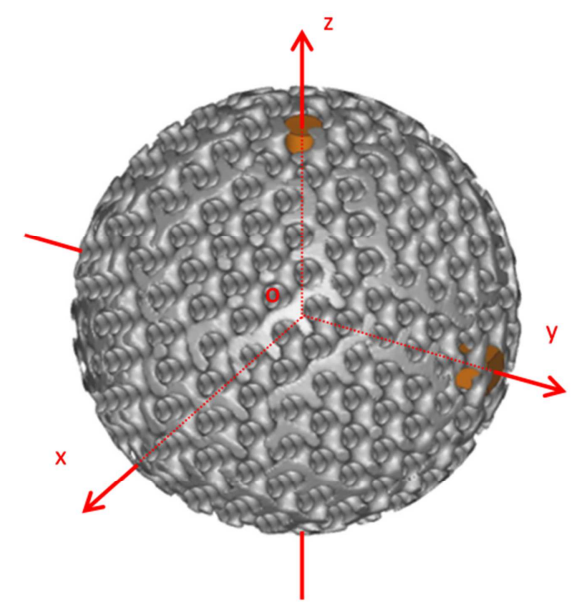

B
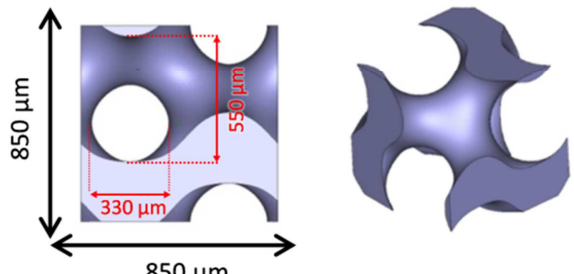

Supplementary Figure S1. Images produced from CAD software of A. The designed bioceramic with the $x-, y$-, and z-axes representing the 3D printer axes and specific visual markers (in orange) serving as orientation references for the geometric data analysis, and B. the gyroid unit cell. 
A

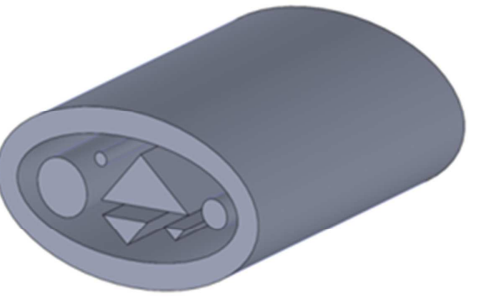

B

C
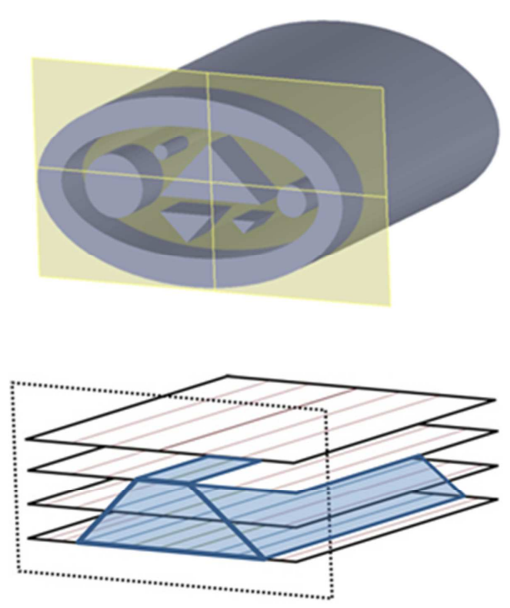

\section{D}

\section{$500 \mu \mathrm{m}$}
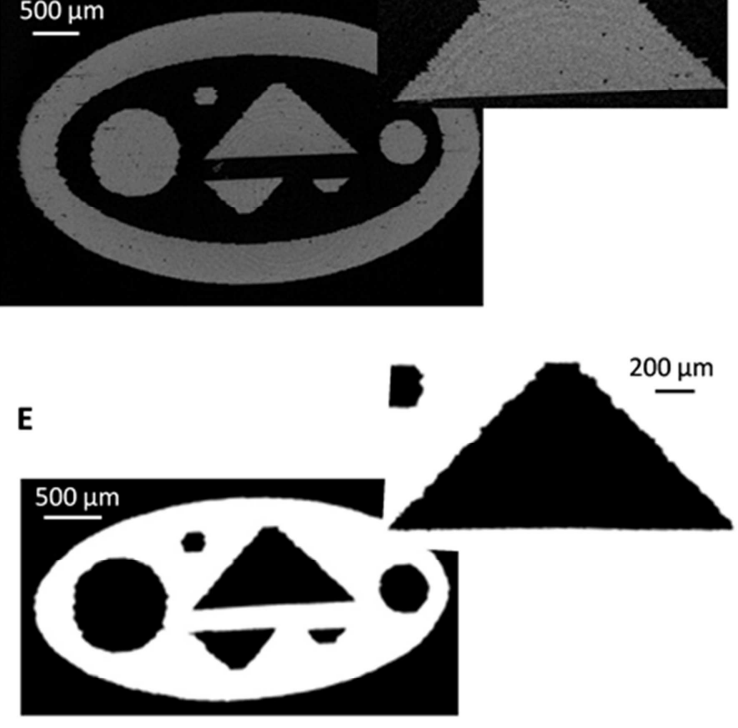

Supplementary Figure S2. Example of the evolution of shape deformation throughout each stage of the bioceramic production process with $A$. the CAD conception of the mold, $B$. the tessellation of the CAD file (.stl export), C. the slicing of the .stl file with respect to a specific orientation of the part (in the current example, mold columns are perpendicular to the z-axis of the printer), D. a cross-section of the produced mold imaged with X-ray tomography and E. a cross section of the resulting bioceramic imaged with X-ray tomography. 